\title{
Delay-Sensitive Resource Allocation for Relay-Aided M2M Communication over LTE-Advanced Networks
}

\author{
Mohammad Reza Mardani ${ }^{\dagger}$, Salman Mohebi ${ }^{\ddagger}$, Behrouz Maham ${ }^{\S}$, and Mehdi Bennis ${ }^{b}$ \\ ${ }^{\dagger}$ School of ECE, College of Engineering, University of Tehran, Iran \\ ${ }^{\ddagger}$ Faculty of New Sciences and Technologies, University of Tehran, Iran \\ ${ }^{\S}$ School of Engineering, Nazarbayev University, Kazakhstan \\ ${ }^{b}$ Centre for Wireless Communication (CWC), University of Oulu, Finland \\ Emails: \{m.mardani,s.mohebi\}@ut.ac.ir, behrouz.maham@nu.edu.kz, bennis@ee.oulu.fi
}

\begin{abstract}
Machine-to-machine (M2M) communications consist of a large number of smart devices that communicate automatically without human intervention. The Third-Generation Partnership Project (3GPP) Long-Term Evolution (LTE) and LTE-Advanced (LTE-A), due to some features such as IP connectivity and scalability, are ready-to-use infrastructures for the M2M communications implementation. In the next generation of cellular networks with M2M devices, radio resource allocation is a major issue. In order to solve the issue, this paper addresses the efficient resource block (RB) allocation problem for different relay- aided cellular and M2M user equipments (UEs) to maximize the end-to-end data rate under different constraints of Single Carrier Frequency Division Multiple Access (SC-FDMA). The proposed solution also satisfies the maximum power budget, the minimum data rate and statistical QoS delay requirements for prioritizing different traffics under total power constraint. Numerical results demonstrate the effectiveness of the proposed scheme.
\end{abstract}

Index Terms-SC-FDMA, Resource allocation, M2M communication, Delay quality-of-service.

\section{INTRODUCTION}

The Third-Generation Partnership Project (3GPP) Long Term Evolution (LTE) standard for uplink multiple access scheme uses Single Carrier Frequency Division Multiple Access (SC-FDMA) [1]. Compared to Orthogonal Frequency Division Multiple Access (OFDMA), SC-FDMA has many benefits such as low Peak-to-Average Power Ratio (PAPR) which improves the efficiency of the transmission power for mobile terminals [2].

Machine-to-machine (M2M) communications provides a way to make connectivity among different machines or devices, independent of human intervention. M2M communications has many different applications such as e-health, smart cities, infrastructure management and monitoring. In the meantime, due to the growing machine type communication (MTC) devices besides currently used human type communication (HTC), it is important to guarantee the quality-ofservice $(\mathrm{QoS})$ requirements for various types of delay sensitive services [3], [4]. Thus, there is a need to intelligent solutions for efficient resource management among all the coexisting MTC and HTC demanded services with respect to their QoS requirements so that none of human-based services are sacrificed.

\section{A. Related Work}

The resource scheduling problem over LTE and LTE-A is discussed in many papers in recent years. A rich survey about scheduling techniques in LTE and LTE-A has been presented in [5] and [6]. The authors of [5] presented a tutorial and a survey about scheduling problems in LTE and LTE-A networks. They also presented an evaluation methodology to compromise the scheduling algorithms. However, in [6], the authors looked to the LTE uplink scheduling problem from an M2M perspective. By considering the M2M communications aspects such as power efficiency, QoS requirements, multi-hop transmission and network scalability, they presented a classification for uplink scheduling techniques over the LTE and LTE-A. The authors in [7] presented a LTE uplink scheduling algorithm that distinguishes between $\mathrm{M} 2 \mathrm{M}$ and $\mathrm{H} 2 \mathrm{H}$ services and applied different scheduling methods for each one. In addition, for M2M services, a two-phase scheduling mechanism based on maximum-utility scheduling and round robin scheduling was presented. Moreover, an algorithm named Iterative Maximum Expansion (IME) is used for scheduling $\mathrm{H} 2 \mathrm{H}$ services. In [8] the impact of massive M2M traffics on the performance of different $\mathrm{H} 2 \mathrm{H}$ services like VoIP, CBR and video over the LTE uplink channels was investigated, when both dynamic and semi-persistent scheduling is used. The authors in [9] and [10] presented a predictive uplink resource allocation scheme for event based M2M application over LTE. The problem of energy conservation in uplink resource and power allocation over LTE-A networks is investigated in [11]. They proposed heuristic methods to reduce the energy consumption of machines while guaranteeing their QoS requirements at the same time. A class based dynamic priority (CBDP) algorithm 
TABLE I: System model and notations

\begin{tabular}{c|c}
\hline Notation & Physical interpretation \\
\hline \hline$K: k \cong\{1, \ldots, K\}$ & Set of traditional cellular UEs (CUEs) \\
\hline$M: m \cong\{1, \ldots, M\}$ & Set of M2M UEs \\
\hline$L: l \cong\{1, \ldots, L\}$ & Set of available RBs \\
\hline$u_{\varsigma}$ & A UE served by relay $\varsigma$ \\
\hline$h_{u_{\varsigma}, 1}^{l}, h_{u_{\varsigma}, 2}^{l}$ & Link gain in first \& second hops over RB $l$, respectively \\
\hline$p_{u_{\varsigma}, 1}^{l}, p_{u_{\varsigma}, 2}^{l}$ & Transmit power in first \& second hops over RB $l$, respectively \\
\hline$\theta_{u_{\varsigma}}^{T}$ & Delay bound for $u_{\varsigma}^{t h}$ UE,$u_{\varsigma} \in\{K \cap M\}$ \\
\hline$x_{u_{\varsigma}}^{l} \in\{0,1\}$ & RB allocation indicator for $u_{\varsigma}$ over $\operatorname{RB} l$ \\
\hline
\end{tabular}

for LTE uplink scheduling with co-existence M2M and $\mathrm{H} 2 \mathrm{H}$ traffics is proposed in [12]. The algorithm considers the delay tolerance and minimum guaranteed bit rate required by communications to achieve the goal of supporting M2M communications with the least impact on $\mathrm{H} 2 \mathrm{H}$ flows. In addition, a variable chunk size based method is proposed in [12] to allocate resource blocks (RBs) to a user. The papers of [13] and [14] introduced a packet scheduling mechanism for LTE networks with M2M communications. The proposed approach uses the system's current information to classify and prioritize data traffics to reduce the impact of $\mathrm{M} 2 \mathrm{M}$ communications on $\mathrm{H} 2 \mathrm{H}$ beside considering the QoS and fairness by adjusting the congestion level. A resource allocation scheme is proposed in [15] that considers the constraints of SC-FDMA for assigning LTE radio resources to device-to-device (D2D) communication more efficiently. The proposed scheme in [15] determines the transmission power of D2D user equipments (DUEs) in order to guarantee certain performance of cellular user equipments (CUEs). Then, this schemes, couples a CUE and a D2D as a resource sharing pair (RSP) and allocate the subchannels based on well-known proportional fairness (PF) scheduling algorithm. A green uplink radio resource allocation schemes for LTE networks is proposed in [16] to efficiently allocate the RBs and transmission power of UEs. The proposed scheme in [16] uses the Opportunistic and Efficient RB Allocation (OEA) algorithm to maximize the aggregate throughput by considering the SC-FDMA constraints. An enhanced version of algorithms, namely, QoS-based OEA is presented to deal with QoS differentiation. To provide the fly wireless communications in the specific geographical area, the paper [17] deployed an unmanned aerial vehicle (UAV) as a flying base station, that considers a co-existence environment with UAV, for downlink data transmission and an underliad D2D communication network. An interval type-2 fuzzy logic approach for resource allocation in LTE is introduced in [18], that enhance the energy consumption and QoS by considering and handling system uncertainties such as noise.

Unlike most of the existing work in LTE-A cellular networks, in this work, we study the advantages of relay for allocating radio resources. Recently, due to the growing number of battery limited $\mathrm{H} 2 \mathrm{H}$ and $\mathrm{M} 2 \mathrm{M}$ devices, which always have not battery replacement option and bandwidth restriction resource, the proper optimization of resources becomes a critical issue. In this paper, we address the problem of resource allocation

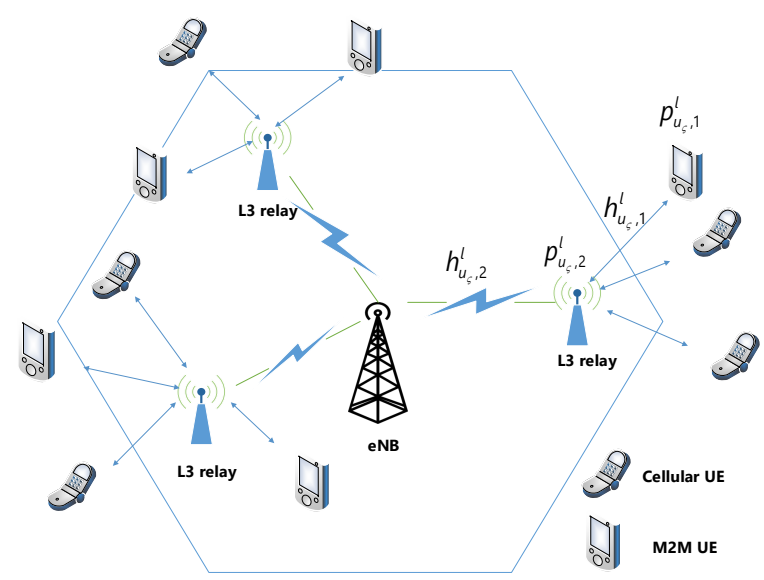

Fig. 1: A multiple relays single cell with multiple $\mathrm{M} 2 \mathrm{M} / \mathrm{H} 2 \mathrm{H}$ coexistence users scenario.

in an LTE-A network with M2M devices.

\section{B. Contributions and Outline}

The main contributions of this paper can be summarized as follows:

- We formulate and obtain globally optimal solution for the problem of RB and power allocation at the relay nodes to analyze the performance of relay-assisted M2M communication. In order to reach the best solution, we formulate the problem considering the delay QoS constraint of different $\mathrm{M} 2 \mathrm{M} / \mathrm{H} 2 \mathrm{H}$ traffics as a priority parameter.

- The radio resource, i.e., RB and transmit power, allocation algorithm is performed with polynomial time complexity analysis at each relay.

The rest of this paper is organized as follows. The system model and assumptions is presented in Section II. In Section III, we formulate the RB and power allocation problem. We propose a distributed algorithm to allocate resources and discuss it's complexity in Section IV. In Section V, we evaluate the performance of results and finally we conclude the paper in Section VI.

\section{SySTEM MOdEL}

\section{A. Network Model}

Fig. 1 shows a single cell with multi-user M2M/H2H coexistence and multiple relay nodes scenario. In addition, some 
important notations and assumptions are defined in Table I. We consider that in order to communicate directly, the cellular user equipment (CUE)-eNB links are undesirable and instead they should employ relays. M2M user equipments (UEs) also need relays due to poor link condition and/or long distance between M2M devices and eNB. Note that we assume that the coherence time of the channel is greater than the Transmission Time Interval (TTI), and $\sigma^{2}=N_{0} B_{R B}$, where $B_{R B}$ is the bandwidth of a RB and $N_{0}$ denotes the thermal noise power per unit of bandwidth in Additive white Gaussian noise (AWGN) channel.

In order to reduce the computation load at the eNB, we use LTE-A Layer 3 (L3) relay ${ }^{1}$ which has capabilities to schedule and allocate system resources among the UEs in the relay node.

\section{B. Achievable Data Rate}

The achievable data rate for each UE (both cellular and M2M users) over RB $l$ in the first and second hop links are represented as:

$$
\begin{aligned}
& R_{u_{\varsigma}, 1}^{l}=B_{R B} \log _{2}\left(1+p_{u_{\varsigma}, 1}^{l} h_{u_{\varsigma}, 1}^{l} / \sigma^{2}\right), u_{\varsigma} \in U=\{K, M\}, \\
& R_{u_{\varsigma}, 2}^{l}=B_{R B} \log _{2}\left(1+p_{u_{\varsigma}, 2}^{l} h_{u_{\varsigma}, 2}^{l} / \sigma^{2}\right), u_{\varsigma} \in U=\{K, M\},
\end{aligned}
$$

respectively. Thus, the overall two hops end-to-end achievable data rate for the $u_{\varsigma}^{t h}$ UE can be calculated as:

$$
R_{u_{\varsigma}}^{l}=\frac{1}{2} \min \left\{R_{u_{\varsigma}, 1}^{l}, R_{u_{\varsigma}, 2}^{l}\right\}
$$

\section{Traffic Prioritizing Weight}

In this work, in order to delay-sensitive protecting, we define a weight $w_{u_{\varsigma}}$ which signifies the user priority in order to ensure the exponent quality of service (QoS) requirement for the $u_{\varsigma}^{t h}$ UE. Stringent and loose exponent QoS requirements are shown by large and small values of $\theta$, respectively. For example, the system can tolerate long delays when $\theta \rightarrow 0$, whereas $\theta \rightarrow \infty$ implies the system cannot tolerate any delay. Finally, the probability of exceeding delay from a maximum bound $\theta_{u_{\varsigma}}^{T}$ is related to $\theta$ according to [20]:

$$
w_{u_{\varsigma}}=e^{-\theta_{u_{\varsigma}}^{T}}
$$

where $\theta_{u_{\varsigma}}^{T}$ is in unit of a symbol duration and denotes the delay bound of each UE. The symbol duration $T_{s}$ is equal to $T_{s}=1 / B$, where $B$ is the system bandwidth.

\section{PROBLEM Formulation}

\section{A. Formulation of the Nominal Resource Allocation Problem}

Let the set of UEs assisted by relay $\varsigma$ is $\Omega_{\varsigma}$ such that $\Omega_{\varsigma} \subset U$ and $\cap_{\varsigma} \Omega_{\varsigma}=\varnothing$. Consider that the maximum allowable transmit power for UE (relay) is $P_{u_{\varsigma}}^{\max }\left(P_{\varsigma}^{\max }\right)$. Hence, the resource allocation problem for each relay $\varsigma$ can be stated as follows:

\footnotetext{
${ }^{1}$ A self-backhaul configuring L3 relay node can operates as an eNB but it has a smaller cell size and uses a lower power to transmission [19].
}

$$
\begin{array}{ll}
\text { (P1) } & \sum_{x_{u_{\varsigma}, p_{u_{\varsigma}, 1}^{l}, p_{u_{\varsigma}, 2}^{l}}^{l}} \\
\text { s.t. } \quad & \sum_{u_{\varsigma}=1}^{\Omega_{\varsigma}} w_{u_{\varsigma}} \sum_{l=1}^{L} x_{u_{\varsigma}}^{l} R_{u_{\varsigma}}^{l} \\
& \sum_{u_{\varsigma}=1}^{\Omega_{\varsigma}} x_{u_{\varsigma}}^{l} \leq 1, \forall l, \\
& \sum_{l=1}^{L} x_{u_{\varsigma}}^{l} P_{u_{\varsigma}, 1}^{l} \leq P_{u_{\varsigma}}^{\max }, \forall u_{\varsigma}, \\
& \sum_{u_{\varsigma}=1}^{\Omega_{\varsigma}} \sum_{l=1}^{L} x_{u_{\varsigma}}^{l} p_{u_{\varsigma}, 2}^{l} \leq P_{\varsigma}^{\max }, \\
& R_{u_{\varsigma}} \geq R_{\min , u_{\varsigma}}, \forall u_{\varsigma}, \\
& p_{u_{\varsigma}, 1}^{l} \geq 0, p_{u_{\varsigma}, 2}^{l} \geq 0, \forall l .
\end{array}
$$

The constraint in (5a) gives each RB to only one UE. The constraints in $(5 b)$ and $(5 c)$ ensure that the transmit power in the first and second hop, respectively, to be bounded by the maximum power thresholds. With the constraint in $(5 \mathrm{~d})$, the minimum QoS requirement $\left(R_{\min }\right)$ is ensured for the CUEs and M2M UEs. Consequently, by using the constraint in $(5 \mathrm{e})$, the non-negativity condition for the transmit power is considered.

From (3), the maximum data rate for UE $u_{\varsigma}$ over RB $l$ is achieved when $p_{u_{\varsigma}, 1}^{l} h_{u_{\varsigma}, 1}^{l}=p_{u_{\varsigma}, 2}^{l} h_{u_{\varsigma}, 2}^{l}$. Hence, with replacing $p_{u_{\varsigma}}^{l} h_{u_{\varsigma}}^{l}=p_{u_{\varsigma}, 1}^{l} h_{u_{\varsigma}, 1}^{l}$, the data rate for UE $u_{\varsigma}$ over RB $l$ can be expressed as: $r_{u_{\varsigma}}^{l}=\frac{1}{2} \log _{2}\left(1+\frac{p_{u_{\varsigma}}^{l} h_{u_{\varsigma}}^{l}}{\sigma^{2}}\right)$. On the other hand, to further simplify the problem, we assumed that $P_{u_{\varsigma}}^{\max }=P_{\varsigma}^{\max }$.

\section{B. Relaxation and Reformulation}

The optimization problem $\mathrm{P} 1$ is computationally intractable due to the fact that it is a mixed-integer non-linear program. In order to tackle such problems, relaxing the constraints is a common approach. Our problem can be relaxed by using the time-sharing factor [21] $x_{u_{\varsigma}}^{l} \in(0,1]$ that causes a RB to be used by only one UE. Moreover, a new variable $\mu_{u_{\varsigma}}^{l}=x_{u_{\varsigma}}^{l} p_{u_{\varsigma}}^{l}$ is introduced which signifies the actual transmit power of UE $u_{\varsigma}$ on RB $l$ [22]. Then, the relaxed upper bound optimization problem can be presented as follows:

$$
\begin{aligned}
\text { (P2) } & \underset{\substack{x_{u_{\varsigma}}^{l}, p_{u_{\varsigma}}^{l} \\
\text { aximize }}}{ } \sum_{u_{\varsigma}=1}^{\Omega_{\varsigma}} w_{u_{\varsigma}} \sum_{l=1}^{L} \frac{1}{2} x_{u_{\varsigma}}^{l} B_{R B} \log _{2}\left(1+\frac{\mu_{u_{\varsigma}}^{l} h_{u_{\varsigma}}^{l}}{x_{u_{\varsigma}}^{l} \sigma^{2}}\right) \\
\text { s.t. } & \sum_{u_{\varsigma}=1}^{\Omega_{\varsigma}} x_{u_{\varsigma}}^{l} \leq 1, \forall l \\
& \sum_{l=1}^{L} \mu_{u_{\varsigma}}^{l} \leq P_{u_{\varsigma}}^{\max }, \forall u_{\varsigma}, \\
& \sum_{l=1}^{L} \frac{1}{2} x_{u_{\varsigma}}^{l} B_{R B} \log _{2}\left(1+\frac{\mu_{u_{\varsigma}}^{l} h_{u_{\varsigma}}^{l}}{x_{u_{\varsigma}}^{l} \sigma^{2}}\right) \geq R_{\min , u_{\varsigma}}, \forall u_{\varsigma}, \\
& \mu_{u_{\varsigma}}^{l} \geq 0, \forall l .
\end{aligned}
$$

The time-sharing condition is satisfied by our optimization problem, and hence, the solution is asymptotically optimal [23]. Since the objective function is convex, the optimization problem P2 is convex, and thus, there exists a unique optimal solution. 


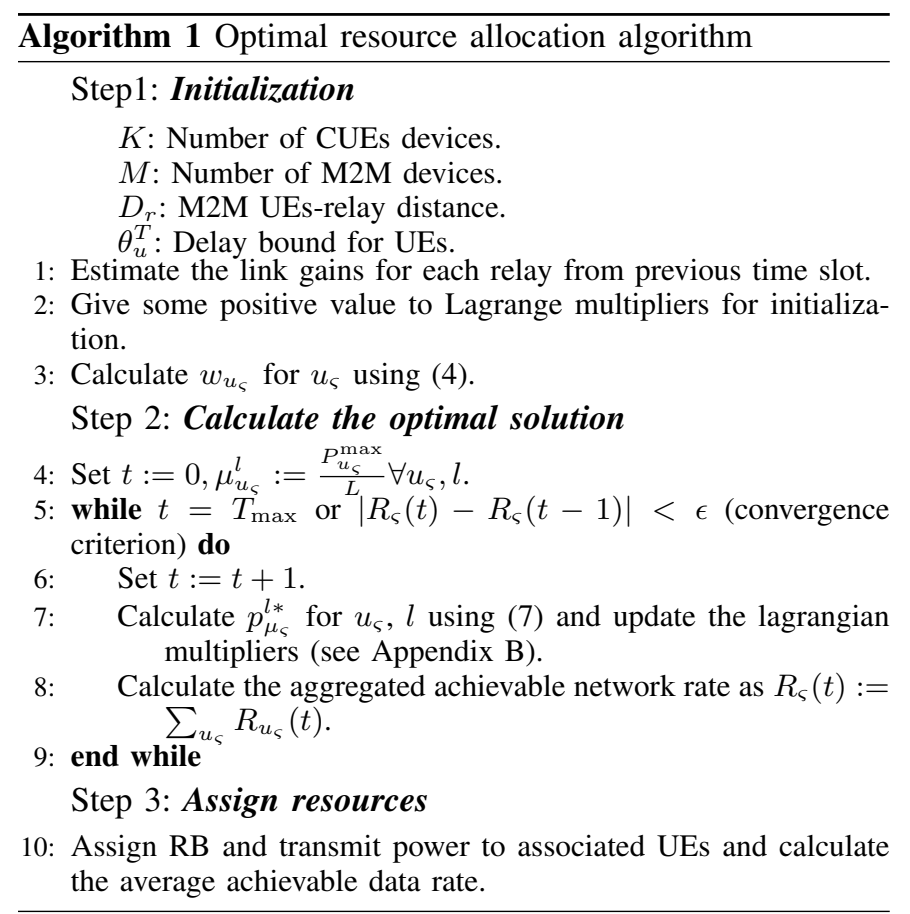

Statement 1: (a) The power allocation for UE $u_{\varsigma}$ on $\mathrm{RB} l$ is given by:

$$
p_{u_{\varsigma}}^{l *}=\frac{\mu_{u_{\varsigma}}^{l *}}{x_{u_{\varsigma}}^{l *}}=\left[\delta_{u_{\varsigma}}^{l}-\frac{\sigma^{2}}{h_{u_{\varsigma}}^{l}}\right]^{+},
$$

where $\delta_{u_{\varsigma}}^{l}=\frac{\frac{1}{2} B_{R B} \frac{\left(w_{u_{\varsigma}}+\phi_{u_{\varsigma}}\right)}{\ln 2}}{\lambda_{u_{\varsigma}}}$ and $q^{+}=\max \{q, 0\}$.

(b) The RB allocation is given as follows:

$$
x_{u_{\varsigma}}^{l *}= \begin{cases}1, & \rho_{l} \leq \xi_{u_{\varsigma}}^{l} \\ 0, & \rho_{l}>\xi_{u_{\varsigma}}^{l}\end{cases}
$$

and

$$
\xi_{u_{\varsigma}}^{l}=\frac{1}{2}\left(w_{u_{\varsigma}}+\phi_{u_{\varsigma}}\right) B_{R B}\left[\log _{2}\left(1+\frac{\mu_{u_{\varsigma}}^{l} h_{u_{\varsigma}}^{l}}{x_{u_{\varsigma}}^{l} \sigma^{2}}\right)-v_{u_{\varsigma}}^{l}\right]
$$

where $v_{u_{\varsigma}}^{l}=\frac{\mu_{u_{\varsigma}}^{l} \gamma_{u_{\varsigma}}^{l}}{\left(x_{u_{\varsigma}}^{l} \sigma^{2}+\mu_{u_{\varsigma}}^{l} \gamma_{u_{\varsigma}}^{l}\right) \ln 2}$ and $\gamma_{u_{\varsigma}}^{l}=\frac{h_{u_{\varsigma}}^{l}}{\sigma^{2}}$.

Proof. See Appendix A.

Proposition 1. A globally optimal solution of the problem P1 can be obtained by $\left(x_{u_{\varsigma}}^{l *}, p_{u_{\varsigma}}^{l *}\right)$.

Proof. The solution $\left(x_{u_{\varsigma}}^{l *}, p_{u_{\varsigma}}^{l *}\right)$, due to the fact that $\mathrm{P} 2$ is a relaxed version of $\mathrm{P} 1$, can gives an upper bound to $\mathrm{P} 1$. On the other hand, a lower bound of P1 can be obtained by P2. It is because the fact that expressions in (7) and (8) are satisfy all P1's constraints due to $x_{u_{\varsigma}}^{l *}$ assures the binary constraints in P1.

\section{Proposed Optimal Algorithm}

\section{A. Joint RB and Power Allocation Algorithm}

The eNB allocates resources to the associated UEs in its coverage area. Algorithm 1 provides the joint RB and power allocation in summary.
Unlike Layer 1 (L1) and Layer 2 (L2) relays in [19], the L3 relays such as an eNB can apply their own scheduling. These relays are able to gather scheduling required information such as the energy consumption at the other relays, link gain information, etc.

\section{B. Complexity of the Proposed Algorithm}

Proposition 2. The proposed algorithm use a gradient-based manner with a small step size to update the variables in (B.1) - (B.3). Number of successive iterations is achieved when the difference of two respectively sum-rate be less than an arbitrary $\varepsilon>0$. Thus, it can be said that the computation complexity at each iteration is a polynomial in $\left|\Omega_{\varsigma}\right|$ and $L$.

Proof. See Appendix C.

\section{Performance Evaluation}

\section{A. Simulation Parameters and Assumptions}

In this section, we evaluate the performance of the proposed resource allocation scheme. Our simulation model and assumptions used for obtaining the numerical results are based on [24]. The channels between each UE and relay and between relay and eNB follow the following path-loss equation, respectively:

$$
P L_{u_{\varsigma}, \varsigma}(d)_{[d B]}=103.8+20.9 \log (d)+L_{s u_{\varsigma}}+10 \log (\varrho),
$$

$$
P L_{\varsigma, e N B}(d)_{[d B]}=100.7+23.7 \log (d)+L_{s \varsigma}+10 \log (\varrho),
$$

where $d$ is the links distance in kilometer, $L_{s u_{\varsigma}}$ and $L_{s \varsigma}$ are log-normal random fading channel and are represented by an exponential variables for modeling shadow fading, respectively; $\varrho$ is the power gain of Rayleigh distributed random variable.

The results are obtained by averaging over 100 realizations of the simulation scenarios i.e., UE locations and link gains. The distance between M2M UE and relay node is denoted by $D_{r}$ which is a simulation parameter in this simulation. The M2M UEs are uniformly distributed in the perimeter of a circle with radius $D_{r}$. For each UE, the delay QoS exponent $\theta_{u_{\varsigma}}^{T}$ constraint is a random value in the interval $\left[10^{-6}, 10^{0}\right]$.

\section{B. Results}

Fig. 2 shows the convergence behavior of the proposed algorithm when $a=0.001$ and $a=0.01$. For convergence, the step size should be selected carefully. We consider the same step size for all the Lagrange multipliers, i.e., for any Lagrange multiplier $\beta$, step size at iteration $\mathrm{t}$ is calculated as $k_{\beta}^{(t)}=\frac{a}{\sqrt{t}}$, where a is a small constant. It is clear from this figure that when a is sufficiently small, the algorithm converges very quickly i.e., in less than 20 iterations, to the optimal solution.

In Fig. 3, we compare the performance of Algorithm 1 under different M2M UE-relay distances when the number of M2M devices increases. The average achievable data rate $R_{\text {ave }}$ for 


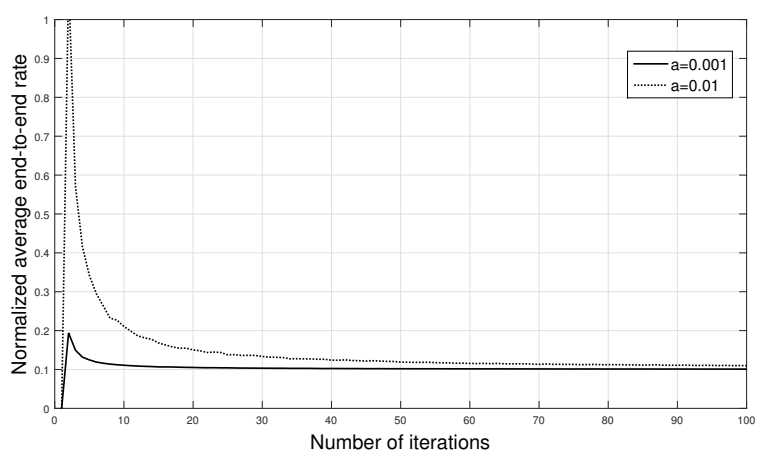

Fig. 2: Convergence behaviour of the proposed algorithm: number of cellular and M2M devices are 15 and $25(|K|=15,|M|=25)$, respectively. The average end-to-end rate is calculated by $\frac{R_{\varsigma}}{\left|U_{\varsigma}\right|}$, the distance of UE from relay, $D_{r}=60$ meter.

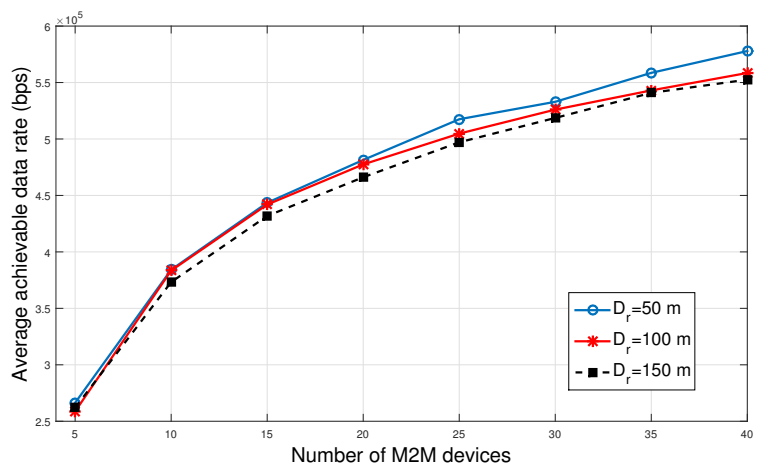

Fig. 3: Performance of the proposed algorithm under different M2M UE-relay distances when the number of cellular UEs is $10(|K|=$ $10)$.

M2M links is calculated as $R_{\text {ave }}=\frac{\sum_{u \in M} R_{u}^{a c h}}{|M|}$, where $R_{u}^{a c h}$ is the achievable data rate for link $u$ and |.| denotes the set cardinality.

Fig. 4 shows the behavior of the proposed resource allocation approach in terms of the achievable aggregated data rate under different number of M2M UEs and different relay-M2M UE distance. As can be seen from this figure, the proposed relaying scheme handles even large relay-M2M UE distances, e.g., $D_{r} \geq 75 \mathrm{~m}$ with considerable achievable rate. To evaluate the performance of our method under moderately and densely situation of network, we vary the number of M2M UEs. It can be seen from Fig. 3 that the achievable rate increases with increasing the number of M2M devices and decreasing the M2M UE-relay distances.

Fig.5 compares the proposed scheme with reference scheme [24] which is ignored traffic priorities effect on simulation results. The graph associated with the proposed algorithm is obtained by averaging of the achievable rate under various delay QoS exponent values. As can be seen from this figure, with increasing the number of M2M UEs, our proposed approach in addition to guarantees the exponent QoS requirements, can performs close to reference scheme with the same complexity.

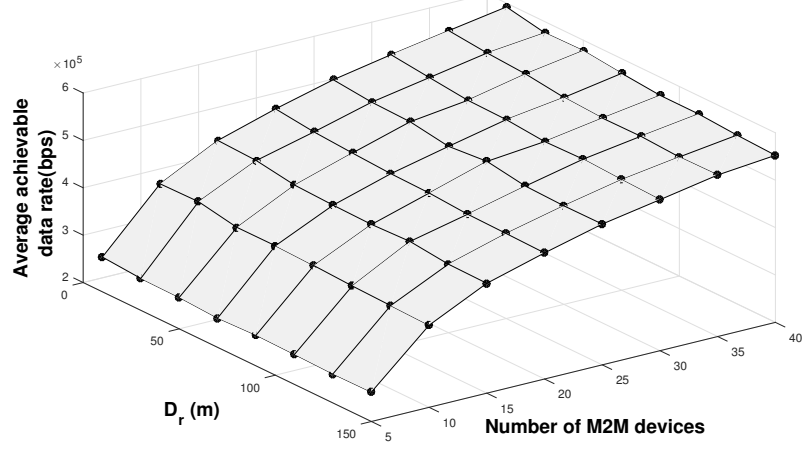

Fig. 4: Achievable aggregated data rate under different number of M2M UEs and different relay-M2M UE distance when the number of cellular UEs is $10(|K|=10)$.

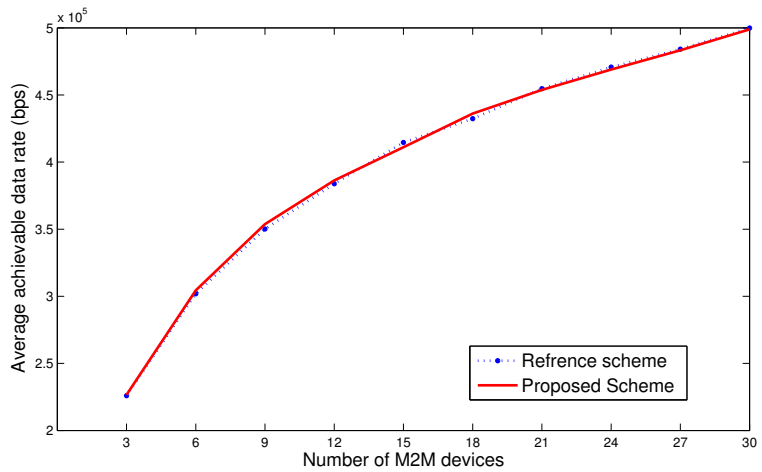

Fig. 5: Comparison of the proposed scheme with refrence scheme. The proposed scheme results by averaging on the various delay QoS exponent values in the interval $\left[10^{-6}, 10^{0}\right]$.

\section{CONCLUSION}

In this paper, we have investigated the optimal delaysensitive resource allocation in LTE networks for relay-aided M2M communication. To allocate radio resources efficiently, we have formulated the resource allocation problem under minimum data rate and statistical delay QoS constraints and we investigated the convexity of the problem. Numerical results have shown that the proposed design is mostly considered as the suitable solution for delay limited applications with constraints on energy consumption of the system.

\section{APPENDIX A}

\section{POWER AND RB Allocation For the Nominal PROBLEM}

We use Karush-Kuhn-Tucker (KKT) Theorem in order to observe the optimality of power allocation for a UE. The Lagrangian function is defined in (A.1), where $\rho, \lambda, \phi$, respectively are the vectors of multipliers associated with assigned resources i.e. RB and transmit power, and individual QoS requirements for UEs. Differentiating (A.1) with respect to $\mu_{u_{\varsigma}}^{l}$ and $x_{u_{\varsigma}}^{l}$, respectively gives the expressions (7) and (8) for power and RB allocation. 


$$
\begin{gathered}
L(X, \mu, \rho, \lambda, \phi)=-\sum_{u_{\varsigma}=1}^{\Omega_{\varsigma}} w_{u_{\varsigma}} \sum_{l=1}^{L} \frac{1}{2} x_{u_{\varsigma}}^{l} B_{R B} \log _{2}\left(1+\frac{\mu_{u_{\varsigma}}^{l} h_{u_{\varsigma}}^{l}}{x_{u_{\varsigma}}^{l} \sigma^{2}}\right) \\
\quad+\sum_{l=1}^{L} \rho_{l}\left(\sum_{u_{\varsigma}=1}^{\Omega_{\varsigma}} x_{u_{\varsigma}}^{l}-1\right)+\sum_{u_{\varsigma}=1}^{\Omega_{\varsigma}} \lambda_{u_{\varsigma}}\left(\sum_{l=1}^{L} \mu_{u_{\varsigma}}^{l}-P_{u_{\varsigma}}^{\max }\right) \\
+\sum_{u_{\varsigma}=1}^{\Omega_{\varsigma}} \phi_{u_{\varsigma}}\left(R_{\min , u_{\varsigma}}-\sum_{l=1}^{L} \frac{1}{2} x_{u_{\varsigma}}^{l} B_{R B} \log _{2}\left(1+\frac{\mu_{u_{\varsigma}}^{l} h_{u_{\varsigma}}^{l}}{x_{u_{\varsigma}}^{l} \sigma^{2}}\right)\right)
\end{gathered}
$$

\section{APPENDIX B}

\section{UPDATE OF VARIABLES AND LAGRANGE MULTIPLIERS}

After that the $p_{u_{\varsigma}}^{l *}$ and $x_{u_{\varsigma}}^{l}$ are obtained, by using expressions (B.1) - (B.3), the variables at the $(t+1)$-th iteration are updated, where $k_{\beta}^{t}$ is the small step size at iteration $t$ for variable $\beta$.

\section{APPENDIX C \\ COMPLEXITY ANALYSIS}

Consider $\left|\Omega_{\varsigma}\right| L$ computations are needed to find the gains and if $T_{\max }$ iterations to be enough in order to convergence of the algorithm, then it can be said that the overall complexity of the applied scheme is $O\left(\left|\Omega_{\varsigma}\right| L+T\left|\Omega_{\varsigma}\right| L\right)$.

Consider $\beta(0)$ is in the interval $\left[0, \beta_{\max }\right]$ for any Lagrange multiplier $\beta$. Then it can be said that $\beta_{\max }$ is upper bound of the distance between $\beta(0)$ and $\beta^{*}$. Also, the upper bound of the distance between the current best objective and the optimum objective at iteration $t$ can be driven by $\frac{\beta_{\max }^{2}+\beta(t)^{2} \sum_{i=1}^{t} k_{\beta}^{(i)^{2}}}{2 \sum_{i=1}^{t} k_{\beta}^{(i)}}$. If the variables updating step size are considered with $k_{\beta}^{(i)}=\frac{a}{\sqrt{i}}$, where $a$ is an arbitrary small constant, then for convergence the bound less than $\varepsilon, O\left(\frac{1}{\varepsilon^{2}}\right)$ iterations are required [25]. Hence, the overall complexity of the proposed algorithm is $O\left(\left|\Omega_{\varsigma}\right| L+\frac{\left|\Omega_{\varsigma}\right| L}{\varepsilon^{2}}\right)$.

$$
\begin{gathered}
\rho_{l}(t+1)=\left[\rho_{l}(t)+k_{\rho_{l}}^{t}\left(\sum_{u_{\varsigma}=1}^{\Omega_{\varsigma}} x_{u_{\varsigma}}^{l}-1\right)\right]^{+}, \\
\lambda_{u_{\varsigma}}(t+1)=\left[\lambda_{u_{\varsigma}}(t)+k_{\lambda_{u_{\varsigma}}}^{t}\left(\sum_{l=1}^{L} \mu_{u_{\varsigma}}^{l}-P_{u_{\varsigma}}^{\max }\right)\right]^{+},
\end{gathered}
$$

$\phi_{u_{\varsigma}}(t+1)=\left[\phi_{u_{\varsigma}}(t)+k_{\phi_{u_{\varsigma}}}^{t}\left(R_{\min , u_{\varsigma}}-\sum_{l=1}^{L} \frac{1}{2} x_{u_{\varsigma}}^{l} B_{R B} \log _{2}\left(1+\frac{\mu_{u_{\varsigma}}^{l} h_{u_{\varsigma}}^{l}}{x_{u_{\varsigma}}^{l} \sigma^{2}}\right)\right]^{+}\right.$

\section{REFERENCES}

[1] H. G. Myung, J. Lim, and D. J. Goodman, "Single carrier FDMA for uplink wireless transmission," Vehicular Technology Magazine, IEEE, vol. 1, no. 3, pp. 30-38, Sep. 2006.

[2] G. Berardinelli, L. Á. M. R. D. Temino, S. Frattasi, M. I. Rahman, and P. Mogensen, "OFDMA vs. SC-FDMA: performance comparison in local area IMT-A scenarios," Wireless Communications, IEEE, vol. 15, no. 5, pp. 64-72, Oct. 2008.

[3] S. Antipolis, "3rd Generation Partnership Project (3GPP), Physical layer aspects for evolved UTRA (Release 7),," 2006. [Online]. Available: http://www.3gpp.org/DynaReport/5814.htm

[4] S. Antipolis, "3rd Generation Partnership Project (3GPP), Service requirements for machine type communications,", 2010. [Online]. Available: http://www.3gpp.org/DynaReport/22368.htm

[5] N. Abu-Ali, A.-E. M. Taha, M. Salah, and H. Hassanein, "Uplink scheduling in LTE and LTE-advanced: tutorial, survey and evaluation framework," IEEE Communications Surveys \& Tutorials, vol. 16, no. 3, pp. 1239-1265, Aug. 2014
[6] M. Mehaseb, Y. Gadallah, A. Elhamy, and H. Elhennawy, "Classification of LTE uplink scheduling techniques: An M2M perspective," IEEE Communications Surveys \& Tutorials, vol. 18, no. 2, pp. 1310-1335, 2016.

[7] S. Zhenqi, Y. Haifeng, C. Xuefen, and L. Hongxia, "Research on uplink scheduling algorithm of massive M2M and H2H services in LTE," in Information and communications technologies (IETICT 2013), IET international conference on. IET, Apr. 2013, pp. 365-369.

[8] T. P. de Andrade, C. A. Astudillo, and N. L. da Fonseca, "Impact of M2M traffic on human-type communication users on the LTE uplink channel," in 2015 7th IEEE Latin-American Conference on Communications (LATINCOM). IEEE, Nov. 2015, pp. 1-6.

[9] J. Brown and J. Y. Khan, "Predictive resource allocation in the LTE uplink for event based M2M applications," in 2013 IEEE International Conference on Communications Workshops (ICC). IEEE, Jun. 2013, pp. 95-100.

[10] J. Brown and J. Y. Khan, "A predictive resource allocation algorithm in the lte uplink for event based M2M applications," IEEE Transactions on Mobile Computing, vol. 14, no. 12, pp. 2433-2446, Dec. 2015.

[11] J. Chen, J. Liang, and Z. Chen, "Energy-efficient uplink radio resource management in LTE-advanced relay networks for internet of things," in 2014 International Wireless Communications and Mobile Computing Conference (IWCMC). IEEE, Aug. 2014, pp. 745-750.

[12] M. K. Giluka, N. Rajoria, A. C. Kulkarni, V. Sathya, and B. R. Tamma, "Class based dynamic priority scheduling for uplink to support M2M communications in LTE," in Internet of Things (WF-IoT), 2014 IEEE World Forum on. IEEE, Mar. 2014, pp. 313-317.

[13] A. M. Maia, M. F. de Castro, and D. Vieira, "A dynamic lte uplink packet scheduler for machine-to-machine communication," in 2014 IEEE 25th Annual International Symposium on Personal, Indoor, and Mobile Radio Communication (PIMRC). IEEE, Sep. 2014, pp. 1609-1614.

[14] A. M. Maia, D. Vieira, M. F. de Castro, and Y. Ghamri-Doudane, "A mechanism for uplink packet scheduler in LTE network in the context of machine-to-machine communication," in 2014 IEEE Global Communications Conference. IEEE, Dec. 2014, pp. 2776-2782.

[15] J. Gu, H. Yoon, J. Lee, S. J. Bae, and M. Y. Chung, "A resource allocation scheme for device-to-device communications using lte-a uplink resources," Pervasive and Mobile Computing, vol. 18, pp. 104-117, Apr. 2015.

[16] F. Z. Kaddour, E. Vivier, L. Mroueh, M. Pischella, and P. Martins, "Green opportunistic and efficient resource block allocation algorithm for lte uplink networks," IEEE Transactions on Vehicular Technology, vol. 64, no. 10, pp. 4537-4550, Oct. 2015.

[17] M. Mozaffari, W. Saad, M. Bennis, and M. Debbah, "Unmanned aerial vehicle with underlaid device-to-device communications: Performance and tradeoffs," IEEE Transactions on Wireless Communications, vol. 15, no. 6, pp. 3949-3963, 2016.

[18] M. R. Mardani, S. Mohebi, and H. Bobarshad, "Robust uplink resource allocation in le networks with $\mathrm{m} 2 \mathrm{~m}$ devices as an infrastructure of internet of things," in Future Internet of Things and Cloud (FiCloud), 2016 IEEE 4th International Conference on. IEEE, 2016, pp. 186-193.

[19] D. I. Kim, W. Choi, H. Seo, and B.-H. Kim, "Partial information relaying and relaying in 3GPP LTE," Cooperative cellular wireless networks, $\mathrm{p}$. 462, Mar. 2011.

[20] D. Wu and R. Negi, "Effective capacity: a wireless link model for support of quality of service," Wireless Communications, IEEE Transactions on, vol. 2, no. 4, pp. 630-643, Jul. 2003.

[21] Z. Shen, J. G. Andrews, and B. L. Evans, "Adaptive resource allocation in multiuser OFDM systems with proportional rate constraints," Wireless Communications, IEEE Transactions on, vol. 4, no. 6, pp. 2726-2737, Nov. 2005.

[22] M. Tao, Y. Liang, and F. Zhang, "Resource allocation for delay differentiated traffic in multiuser OFDM systems," Wireless Communications, IEEE Transactions on, vol. 7, no. 6, pp. 2190-2201, Jun. 2008.

[23] W. Yu and R. Lui, "Dual methods for nonconvex spectrum optimization of multicarrier systems," Communications, IEEE Transactions on, vol. 54, no. 7, pp. 1310-1322, Jul. 2006.

[24] M. Hasan, E. Hossain, and D. I. Kim, "Resource allocation under channel uncertainties for relay-aided device-to-device communication underlaying LTE-A cellular networks," Wireless Communications, IEEE Transactions on, vol. 13, no. 4, pp. 2322-2338, Apr. 2014.

[25] S. Boyd, L. Xiao, and A. Mutapcic, "Subgradient methods," lecture notes of EE392o, Stanford University, Autumn Quarter, vol. 2004, pp. 2004-2005, Oct. 2003. 